\title{
The soft explosive model of placental mammal evolution
}

\author{
Matthew J. Phillips ${ }^{*}$ (D) and Carmelo Fruciano
}

\begin{abstract}
Background: Recent molecular dating estimates for placental mammals echo fossil inferences for an explosive interordinal diversification, but typically place this event some 10-20 million years earlier than the Paleocene fossils, among apparently more "primitive" mammal faunas.

Results: However, current models of molecular evolution do not adequately account for parallel rate changes, and result in dramatic divergence underestimates for large, long-lived mammals such as whales and hominids. Calibrating among these taxa shifts the rate model errors deeper in the tree, inflating interordinal divergence estimates. We employ simulations based on empirical rate variation, which show that this "error-shift inflation" can explain previous molecular dating overestimates relative to fossil inferences. Molecular dating accuracy is substantially improved in the simulations by focusing on calibrations for taxa that retain plesiomorphic life-history characteristics. Applying this strategy to the empirical data favours the soft explosive model of placental evolution, in line with traditional palaeontological interpretations - a few Cretaceous placental lineages give rise to a rapid interordinal diversification following the $66 \mathrm{Ma}$ CretaceousPaleogene boundary mass extinction.

Conclusions: Our soft explosive model for the diversification of placental mammals brings into agreement previously incongruous molecular, fossil, and ancestral life history estimates, and closely aligns with a growing consensus for a similar model for bird evolution. We show that recent criticism of the soft explosive model relies on ignoring both experimental controls and statistical confidence, as well as misrepresentation, and inconsistent interpretations of morphological phylogeny. More generally, we suggest that the evolutionary properties of adaptive radiations may leave current molecular dating methods susceptible to overestimating the timing of major diversification events.
\end{abstract}

Keywords: Cretaceous-Paleogene boundary, Fossil calibration, Life history, Molecular dating, Placentalia

\section{Background}

Molecular and palaeontological analyses of placental mammals both identify an interordinal diversification spike, in which the stem lineages of nearly all 18 modern orders (e.g. primates, rodents) originated over a period of just a few million years (Ma). However, most molecular dating estimates (e.g. [1-3]) for this diversification are 10-20 Ma older than observed in the fossil record $[4,5]$. The extraordinary fossil record surge for eutherians (crown placentals and their extinct stem relatives) follows the $66 \mathrm{Ma}$ Cretaceous-Paleogene boundary (KPg) mass extinction event (Fig. 1). This fossil record diversification also manifests as a taxonomic phase change, with eutherians as a percentage of new mammal

\footnotetext{
* Correspondence: m9.phillips@qut.edu.au

School of Earth, Environmental and Biological Sciences, Queensland University of Technology, Brisbane, Australia
}

species appearances increasing from an average of $27 \%$ during the Campanian and Maastrichtian, to $84 \%$ during the Paleocene.

If the older molecular dates for the interordinal diversification are instead correct, then this most profound event in placental history leaves no discernible trace in the fossil record (Fig. 1). This is especially perplexing, because ancestral area reconstruction [6] places this proposed $\sim 75-85$ Ma molecular radiation (including stem members of all 11 Northern Hemisphere orders) right alongside the best Late Cretaceous fossil faunas in Eurasia and North America. It is similarly incongruous that during the diversity surge in the placental fossil record following the KPg mass extinction $[7,8]$ those same molecular timetrees instead imply stable or even declining diversification [2, 9]. Springer et al.'s [10] new tree does place several additional primate and rodent divergences

(c) The Author(s). 2018 Open Access This article is distributed under the terms of the Creative Commons Attribution 4.0 International License (http://creativecommons.org/licenses/by/4.0/), which permits unrestricted use, distribution, and 


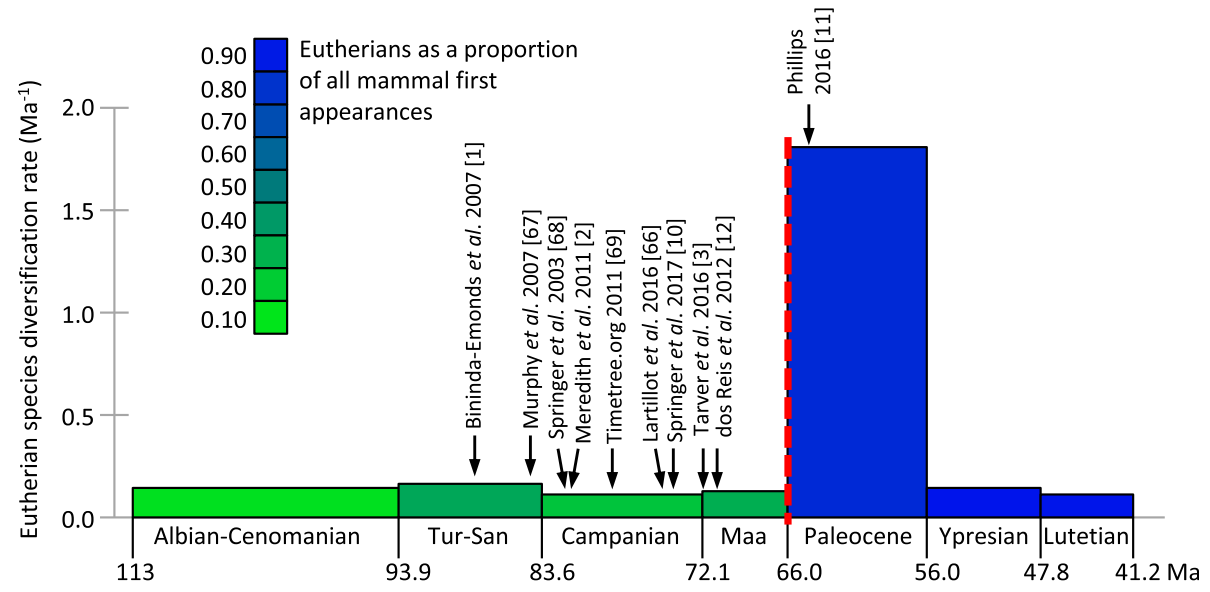

Fig. 1 Eutherian fossil record species diversification rate. Data points are calculated as new appearances/Ma (scaled by species richness in the previous time bin, a proxy for starting species richness). Barremian-Aptian provides the previous time bin for Albian-Cenomanian. The green-blue colour shading indicates the proportion of eutherians among mammal first appearances for each time bin. Referenced arrows indicate molecular dating estimates for the temporal midpoint of the placental interordinal diversification, including for Laurasiatheria, Euarchontoglires and Afrotheria (See Additional file 2: Table S2). The KPg boundary is shown as red dashes. Tur-San, Turonian to Santonian; Maa, Maastrichtian [1-3], [10-12] [66-69]

close to the KPg relative to [2], but this may be an artefact of adding maximum bounds at the KPg for these clades to bump up against.

Phillips [11] recently presented evidence for two methodological contributors to molecular dates overestimating early divergences among placentals: (1) Molecular clocks over-smooth parallel decelerations in evolutionary rates among large, long-lived mammals. This results in several-fold divergence underestimates in groups such as whales and seacows, for which calibration to correct these clade ages simply transfers the underlying rate error stemwards, and inflates divergence estimates deeper in the tree. (2) Such "error-shift inflation" is further facilitated by asymmetry in calibration priors between minimum bounds that are highly speculative, and maximum bounds that are too conservative to buffer against rate misspecification or erroneous minimum bounds at other nodes.

Phillips [11] sought to ameliorate error-shift inflation in two steps. The first reduced the impact of oversmoothed, parallel rate decelerations on dates deeper in the tree, by employing dos Reis et al.'s [12] calibration scheme - which includes fewer constraints among large, long-lived taxa than does Meredith et al.'s [2] scheme. The second step reduced asymmetry in fossil calibration priors, by revising overly conservative maximum bounds in line with best practices [13], so as to enhance the capacity of the calibration scheme to buffer against rate errors. The revised calibration scheme was then used to reanalyse Meredith et al.'s [2] 26-locus dataset for 169 taxa, and resulted in molecular dates that closely matched long-held fossil record expectations [14-16]. We refer to this as the "soft explosive" model of placental evolution; a few Cretaceous placental lineages seed the massive interordinal diversification spike that follows the KPg extinction event. In contrast, the "hard explosive" model $[4,5]$ places both the placental origin and initial radiation near or after the KPg.

Although the soft explosive model brings agreement between molecular and fossil inference of placental evolution, it has recently been criticized by Springer et al. [10] on two grounds. The first criticism is that Phillips [11] erroneously dragged divergences younger by "eliminating calibrations in large-bodied/long lifespan clades" without deleting those taxa. This claim is false. Phillips [11] maintained each of dos Reis et al.'s [12] calibrations that were placed in large-bodied/long lifespan clades. Springer et al.'s [10] argument was also based on an analysis in which they deleted large, long-lived taxa, and found that most supraordinal divergences increased by 8-10 Ma relative to Phillips [11]. However, Springer et al. [10] failed to control for calibration, and it is not their taxon deletion, but their inclusion of poorly supported calibrations that drives the divergence estimates older (as discussed below; also see Additional file 1). Indeed, when we repeat their taxon deletion, but maintain the original calibration scheme of Phillips [11], the divergence estimates again support the soft explosive model (dR32 analysis, Table 1C).

Springer et al.'s [10] second criticism of the soft explosive model was that many divergences post-date the earliest fossil evidence for the clade, thus implying the existence of fossil "zombie" lineages. We will show that this criticism is based on misrepresentation, false precision in their interpretation of molecular dates, and overconfidence in poorly resolved fossil relationships. 
Table 1 Mean MCMCtree posterior estimates (Ma)

\begin{tabular}{|c|c|c|c|c|c|c|}
\hline \multirow[t]{3}{*}{ Clades } & \multirow{3}{*}{$\begin{array}{l}169 \text { taxa: } \\
\text { calibrating } \\
\text { mostly } \\
\text { "ancestral } \\
\text { rates" clades } \\
\text { (A) Phillips [11] }\end{array}$} & \multicolumn{5}{|c|}{122 taxa: large, long-lived taxa deleted } \\
\hline & & \multirow{2}{*}{$\begin{array}{l}\text { Alternative calibration } \\
\text { (B) Springer et al. [10] }\end{array}$} & \multirow{2}{*}{$\begin{array}{l}\text { Calibration from Phillips [11] } \\
\text { (C) dR } 32\end{array}$} & \multicolumn{3}{|c|}{ Additional well-supported calibrations } \\
\hline & & & & (D) dR40 & (E) dR40 ind $95 \% \mathrm{Cl}$ & (F) dR40 auto $95 \% \mathrm{C}$ \\
\hline Placentalia & 78.2 & 92.4 & 76.1 & 78.7 & $76.3-80.9$ & $76.6-81.0$ \\
\hline Laurasiatheria & 66.8 & 75.8 & 65.5 & 66.6 & $64.3-67.7$ & $65.4-69.0$ \\
\hline Scrotifera & 64.4 & 74.0 & 64.1 & 64.9 & $62.4-66.0$ & $63.7-67.4$ \\
\hline Fereuungulata & 64.3 & 73.4 & 63.9 & 64.3 & $61.9-65.5$ & $63.2-66.9$ \\
\hline Ferae & 62.3 & 70.7 & 62.7 & 61.6 & $59.0-62.8$ & $60.7-64.6$ \\
\hline Euarchontoglires & 67.7 & 76.7 & 66.3 & 67.9 & $66.3-69.3$ & $66.5-69.3$ \\
\hline Archonta & 67.3 & $76.2^{\mathrm{a}}$ & 66.0 & 67.1 & $65.3-68.5$ & $65.8-68.5$ \\
\hline Primatomorpha & 67.1 & 75.2 & 65.7 & 66.2 & $64.4-67.6$ & $64.9-67.5$ \\
\hline Glires & 65.7 & 73.7 & 64.3 & 65.5 & $64.2-67.1$ & $63.9-66.6$ \\
\hline Afrotheria & 59.4 & 76.6 & 61.2 & 63.0 & $61.3-66.6$ & $59.9-64.7$ \\
\hline Afroinsectivora & 57.7 & 73.5 & 59.3 & 60.3 & $58.7-63.7$ & $57.4-62.2$ \\
\hline
\end{tabular}

Dates refer to the crown ages of placental mammals and ten of the twelve interordinal clades identified by Meredith et al. [2] among the primary placental diversification spike. The two other clades, Afroinsectiphilia and Zooamata are excluded in the 122 taxon data sets based on Springer et al.'s [10] deletion of large, long-lived taxa. The average of independent (ind) and autocorrelated (auto) rates model divergence dates are provided for A-D, with $95 \%$ credible intervals provided for the dR40 timetrees for both the independent rates (E) and autocorrelated rates (F) analyses. (A) divergences from Phillips ([11], Fig. 4d), (B) Springer et al.'s [10] 122-taxon reanalysis of Phillips [11] with a new calibration scheme, (C) our analysis of the 122-taxon data set focusing on Phillips' [11] calibrations, and then adding eight further calibrations (D-F, see Additional file 3)

${ }^{a}$ In Springer et al. [10] Scandentia groups with Glires, hence, we show the age of that grouping instead of Archonta (Scandentia/Primatomorphia)

One point of agreement in the debate over the timescale of placental evolution is that calibrating among large, long-lived taxa results in older age estimates for the root and interordinal divergences $[2,10,11]$. Nevertheless, attempting to understand and quantify the contribution from error-shift inflation may be confounded by variation in calibration precision (how closely fossil calibrations match true divergences) - which may differ between the deleted large, long-lived calibrations and the remaining calibrations. Here we use simulations based on empirical estimates of molecular rate variation among placentals to control for calibration accuracy and precision, and to better understand the influence of error-shift inflation.

Considered together, our simulation study and new molecular dates based on revised fossil calibrations for the datasets of Meredith et al. [2] and Liu et al. [17] provide strong support for the soft explosive model of placental diversification. Moreover, previous, older molecular dates are explained as artefacts of errors in both calibration and modelling rate variation across the tree. In turn, the younger KPg diversification allows us to revise Romiguier et al.'s [18] surprising molecular inference of early placental life history traits. More generally, there is a wider pattern of conflict between molecular dates and fossil evidence for the timing of major diversifications, such as for birds [19, 20], flowering plants [21-23] and the Cambrian explosion [24]. We discuss the possibility that major adaptive radiations could be particularly susceptible to error-shift inflation, resulting in molecular divergence overestimates.

\section{Results and discussion}

Simulated rate deceleration among large, long-lived taxa mimics observed molecular dating errors

To control for calibration and isolate the behaviour of error-shift inflation, we simulated molecular data on a phylogeny of given age (Fig. 2a) that is simplified from the proposed mammalian timetree of Phillips (Figure five in [11]). We simulate "placentals" originating at $80 \mathrm{Ma}$, then splitting into two 66 Ma superorders (e.g. "Laurasiatheria" and "Afrotheria"), which each give rise to two $33 \mathrm{Ma}$ calibrated clades. In the first set of simulations the branch rates are randomly drawn from a lognormal distribution modelled on inferred rates from Phillips [11] for small to mid-sized mammals $(<30 \mathrm{~kg}$ adult body mass, $<40$ years maximum longevity). Relaxed molecular clock dating in MCMCtree $[25,26]$ accurately reconstructs all node heights under this simple distribution of rates across the tree (Fig. 2c-e, light grey bars). However, when we simulate a parallel rate deceleration reminiscent of whales or seacows for just one of the $33 \mathrm{Ma}$ calibrated nodes in each superorder, the MCMCtree reconstructions reveal extreme error-shift inflation. Average estimates for the $66 \mathrm{Ma}$ superorders were inflated to $80.5 \mathrm{Ma}$, and the $80 \mathrm{Ma}$ placental root was inflated to 107.9 Ma. In each case the 95\% CIs are fully older than the simulated dates. These inflated divergences closely mimic recent molecular dates for placental mammals.

Our simulations also reproduce the empirical pattern of extreme dating underestimation for large, long-lived clades for when they are not calibrated. In particular, the 


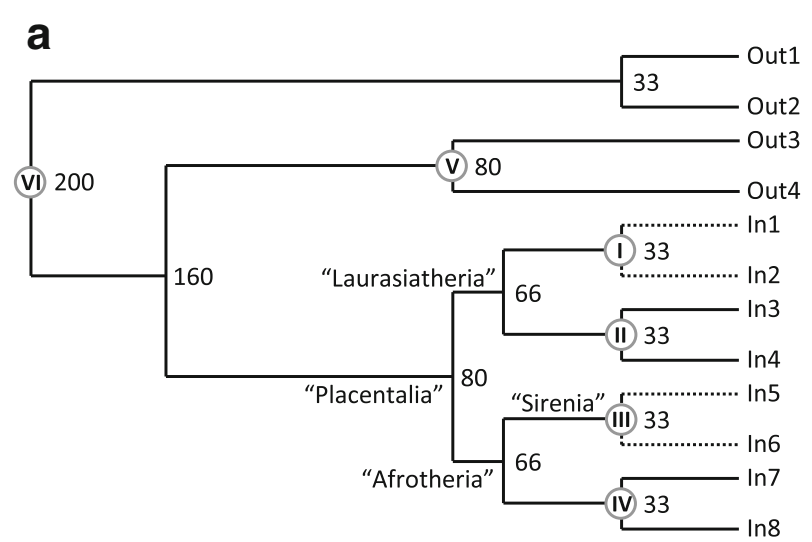

b

\begin{tabular}{|ccc|}
\hline \multicolumn{3}{|c|}{ Calibration bound schemes (Ma) } \\
Node & $\begin{array}{c}\text { Full } \\
\text { calibration }\end{array}$ & $\begin{array}{c}\text { Ancestral } \\
\text { rate nodes }\end{array}$ \\
\hline I & $28-38$ & - \\
\hline II & $28-38$ & $28-38$ \\
\hline III & $28-38$ & - \\
\hline IV & $28-38$ & $28-38$ \\
\hline V & $60-100$ & $60-100$ \\
\hline VI & $180-220$ & $180-220$ \\
\hline & & \\
\hline
\end{tabular}
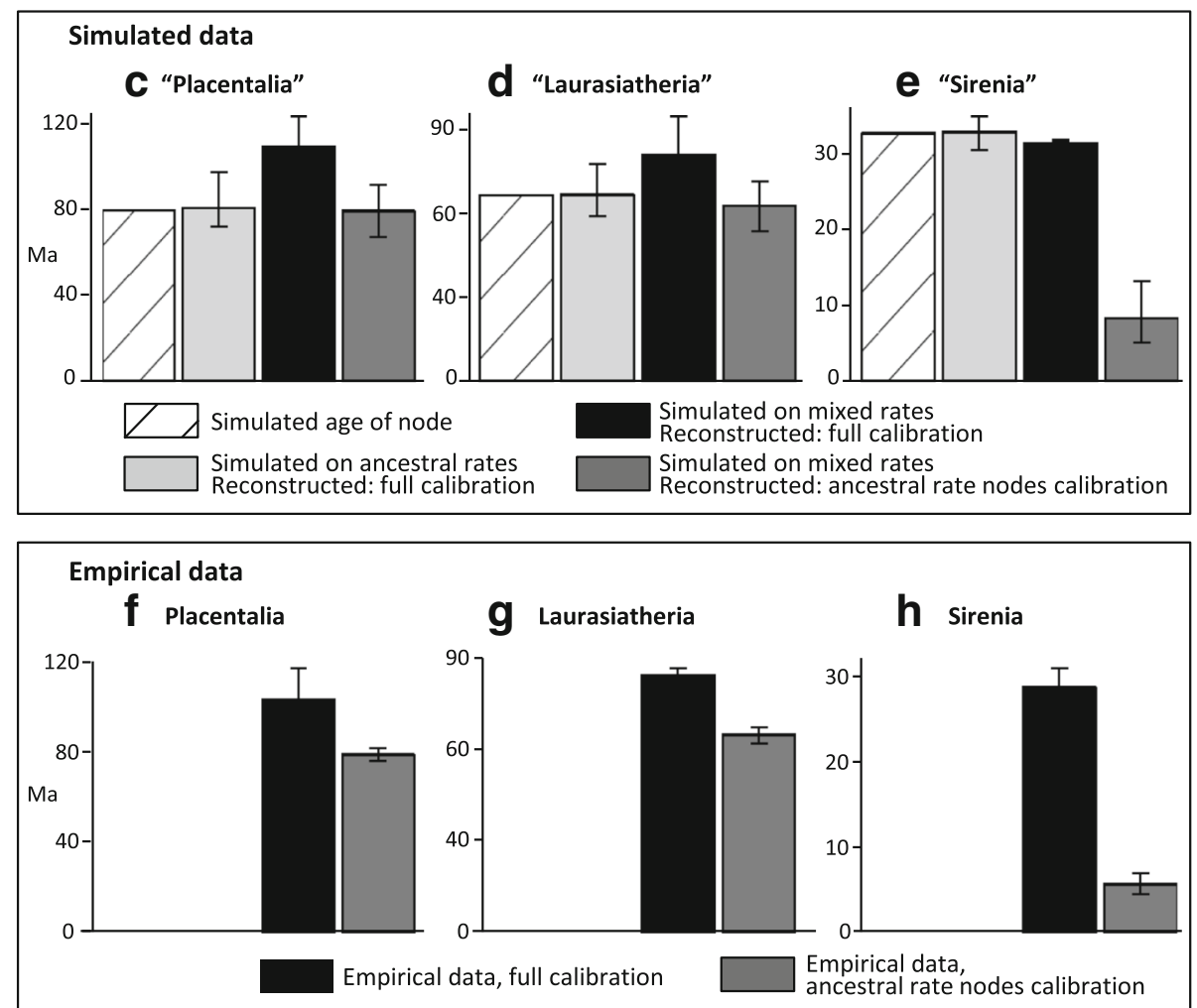

Fig. 2 Simulating error-shift inflation of deep placental divergences, and amelioration by excluding calibrations among large, long-lived clades. a. Dated tree on which 20,000 bp DNA sequences were simulated under two rate schemes, "ancestral rates" with all branch rates drawn from a single lognormal distribution (see Methods) and "mixed rates" with the rate drawn from that same distribution, except divided by 5 for the daughter lineages of nodes I and III. b. Soft bound calibrations on nodes I-VI under alternative MCMCtree reconstructions. Date estimates and 95\% Cls for simulated clades c "Placentalia" $\mathbf{d}$ "Laurasiatheria" and e "Sirenia" are shown for the "ancestral rates" simulation (light grey), and for the "mixed rates" simulation with either full calibration (black) or calibration only on ancestral rates clades (dark grey). Corresponding date estimates from Phillips ([1 1], Fig. 4c,d) on the empirical data are shown for $\mathbf{f}$ Placentalia, $\mathbf{g}$ Laurasiatheria and $\mathbf{h}$ Sirenia, with full calibration (black) and largely focusing on ancestral rates clades (dark grey)

low rate clades simulated as $33 \mathrm{Ma}$ are reconstructed by MCMCtree with a mean age of 8.5 Ma, almost as extreme as the empirical pattern for seacow origins falling from $\sim 28 \mathrm{Ma}$ to $5.7 \mathrm{Ma}$ when uncalibrated (Fig. 2e,h). Importantly though, excluding calibrations among the low rate (large, long-lived) clades allows accurate inference of divergence dates deeper in the tree, returning reconstructions close to the simulated ages (Fig. 2c,d "mixed rates ancestral calibrations" - dark grey bars). It is remarkable how closely the pattern of uncalibrating large, long-lived taxa to overcome the simulated error-shift inflation (Fig. 2c-e) mirrors the empirical pattern for placental mammals (Fig. 2f-h). Thus, our simulations, which are based on empirical rate variation, show that error-shift inflation associated with parallel rate deceleration among large, long-lived placentals can 
explain the proposed overestimation of interordinal divergences among molecular dating analyses.

\section{Conjuring up "zombie" lineages}

Lane et al. [27] coined the term "zombie lineage" for the extension of a taxon's survival beyond their last fossil appearance. Springer et al. [10] re-purposed the term for molecular divergences that are younger than minimum ages implied by fossil records, and claim that Phillips' [11] "preferred timetree" includes 61 (of 136) internal placental nodes that are younger than first fossil appearances, thus resulting in "zombie" lineages. Springer et al.'s [10] claim is based on a series of misrepresentations, which are best appreciated by first understanding how Phillips' [11] timetree was constructed. Phillips [11] recognised that calibrating large, long-lived taxa in the tree of more plesiomorphic mammals erroneously inflates interordinal divergences (also shown here with simulations, Fig. 2c,d "full calibration" black bars), whereas not calibrating among these taxa underestimates their own family-level divergences (Fig. 2e "mixed rates ancestral calibrations" - dark grey bar). Phillips [11] addressed this challenge in two steps. The first step inferred divergences with dos Reis et al.'s [12] calibrations, most of which are set among taxa with plesiomorphic life-history (tree 1, Fig. $4 \mathrm{~d}$ in [11]). The final timetree (tree 2, Figure five in [11]) was then inferred with more calibrations added among large, long-lived taxa, but with maximum bounds on several superordinal clades based on broad agreement between tree 1 and fossil records for major diversification following the KPg (and also noting that multi-lineage diversifications should provide more robust markers in the fossil record than individual nodes).

Springer et al.'s [10] misrepresentation begins by overlooking Phillips' [11] discussion of uncalibrated divergences among large, long-lived taxa being underestimated in tree 1 , and falsely claiming tree 1 to be Phillips' [11] "preferred tree". They then ignore the final timetree with those taxa calibrated (tree 2), which Phillips [11] used for final inference of molecular rates, and instead, Springer et al. [10] set up the tree 1 dates as a straw man for comparison with fossil dates.

A careful examination of the (actually) 62 nodes that Springer et al. [10] tabled as postdating proposed fossil dates reveals that 40 involve clades of large/long-lived taxa. These underestimates follow directly from the aims for tree 1 , which were to reveal the extent of date underestimation among large, long-lived clades and to isolate the interordinal nodes from error-shift inflation that would result from those large, long-lived clades being calibrated (as our simulation study confirms, Fig. 2). Springer et al. [10] perhaps agree, and deleted all 40 of those large, long-lived clades for their analysis.

The more concerning claim that Phillips [11] underestimated the age of 22 clades that retain apparently more plesiomorphic life history traits is illusory, created from false precision. Springer et al. [10] exaggerate disagreement here by treating those molecular dates as errorless, and by ignoring uncertainty in the phylogenetic attribution of reference fossils for minimum bounds. If instead we undertake the usual practices of basing fossil minimum dates on phylogenetically well-supported fossils, and considering Bayesian molecular divergences as 95\% credible intervals (CIs), then the discrepancy vanishes for 20 of the 22 clades (Additional file 1). Moreover, Phillips' [11] final molecular dates (tree 2) place the 95\% CIs for the two remaining clades, Musteloidea (28.5-30.7 Ma) and Feliformia (30.3-35.3 Ma) entirely older than their respectively proposed fossil minima of $24.8 \mathrm{Ma}$ and $28.1 \mathrm{Ma}$. The tree 2 analysis was primarily designed to test rate variation hypotheses, and still retains some dubious fossil calibrations from Meredith et al. [2]. But it is notable that our primary dating estimates in this study are also consistent with both of these proposed fossil minima (Additional file 2, 122-taxon dR40 trees). Thus, Springer et al.'s [10] claim of "zombie" lineages among smaller, shorter lived taxa is unfounded.

\section{Dating the evolution of placental mammals}

We have updated our calibration set to allow for eight additional well-supported calibrations (Additional file 3) that were not employed by dos Reis et al. [12], but include several that Springer et al.'s [10] list of "zombie" lineages implied would increase our divergence estimates. This lifts the number of calibration priors to 40 for the same 122 taxa with apparently plesiomorphic life histories that were employed by Springer et al. [10]. The resulting MCMCtree timetree (dR40, see Table 1D) provides very similar divergence estimates to our previous calibration schemes (Table 1A,C). The most profound diversification in placental mammal history again falls across or closely follows the KPg boundary (see Fig. 3a), including for the basal radiations of all three major superorders (Laurasiatheria, Afrotheria and Euarchontoglires).

To isolate the source of the differences between our dates and Springer et al.s [10] dates we identified poorly-vetted reference fossils that they used to define seven placental calibration minima that are older than our dR40 molecular estimates (Table 2). In several cases the temporal difference is so minor $(1.5-2.8 \mathrm{Ma})$ as to have little impact deeper in the tree. However, four of Springer et al.'s [10] fossil minima are strikingly older than our molecular estimates, and reveal breathtaking inconsistency in how these authors treat morphological phylogenetic evidence. For example, Springer et al. [10, 28, 29] express valid cautions, and are highly critical of morphological phylogeny, even for well-sampled modern or Mesozoic eutherians that are analysed within objective, statistical 


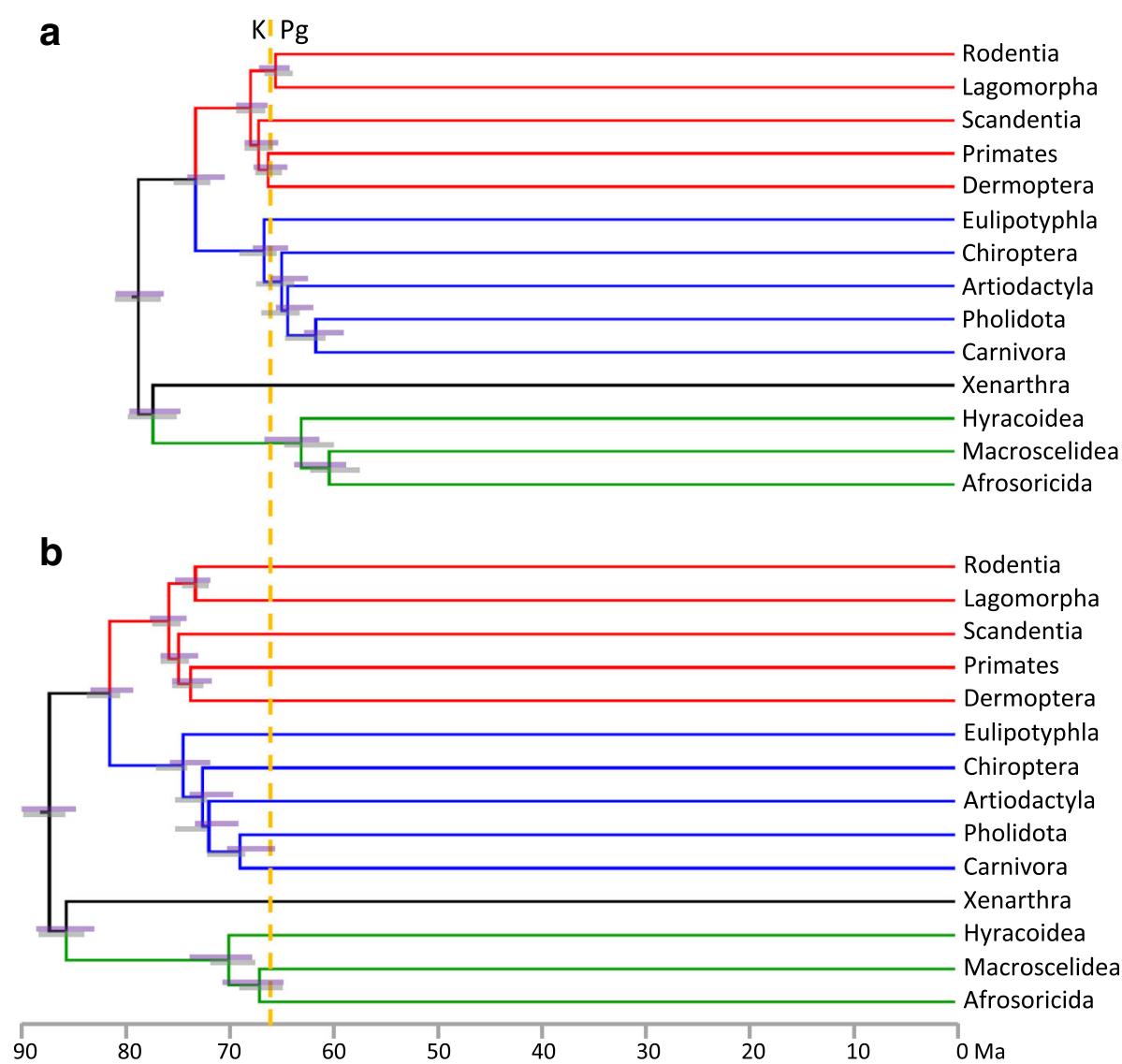

Fig. 3 Placental mammal ordinal-level timetrees on the 122-taxon dataset for which large, long-lived taxa are excluded. Node heights are averaged over MCMCtree independent and autocorrelated rates analyses, with 95\% Cls shown for analyses under independent rates (purple bars) and autocorrelated rates (grey bars). a. using our dR40 calibration set. b. Adding additional poorly-vetted calibrations for Lorisiformes, Lagomorpha, Emballonuroidea and Erinaceidae-Soricidae, and with maximum bounds for basal Primates, Rodentia and Chiroptera increased, following Springer et al. [10]. Substituting in these "dR40 springer" calibrations inflates the midpoint for the primary placental interordinal diversification from 64.5 Ma to 72.2 Ma

frameworks. Yet, when it comes to calibration, Springer et al. [10] accept reference fossils based on highly fragmentary material, unverified by any formal phylogenetic analyses (matrix-based or otherwise) or that are contradicted by such analyses (see Additional file 1) - then employ these fossils as minimum bounds with $97.5 \%$ or indeed, preferentially with $100 \%$ prior probability.

The four poorly-vetted fossils that substantially conflict with our molecular dates are:

(1) The 38 Ma Saharagalago (calibrating Lorisiformes) is known from just two molars. Phillips (Figure two in [11]) showed this calibration to be an extreme outlier for apparent dating error (or rate distortion). The most likely explanation is that Saharagalago (and Karanisia from the same locality) falls outside Lorisiformes, as two recent phylogenetic analyses found [30, 31].

(2) The $~ 53 \mathrm{Ma}$ 'Vastan' ankle bones (calibrating Lagomorpha) were found by Rose et al. [32] to group with Oryctolagus to the exclusion of other rabbits and hares. If true, these ankle bones would implausibly pre-date molecular dating expectations for the Oryctolagus-Sylvilagus divergence by $\sim 5$ fold [33]. However, Rose et al. [32] did not consider sampling error and noted that the morphological signal may be confounded by functional similarities. Zhang et al.s [34] $\mu \mathrm{CT}$ scans have since shown that a key character, the calcaneal canal, is also present in stem lagomorphs. Our re-analysis of Rose et al. [32] excluding the calcaneal canal character places the Vastan ankle bones outside crown lagomorphs, although their affinities remain statistically unresolved (Additional file 1: Figure S1).

(3) The $\sim 47$ Ma bat, Tachypteron (calibrating Emballonuroidea) was assigned by Storch et al. [35] only on the basis of similarities, and within a framework that considered emballonurids as sister to rhinolophoids. These two groups are now known to fall on opposite sides of the chiropteran tree [36], 
Table 2 Springer et al.'s [10] placental calibration minima that are older than our 95\% Cls for both the independent and autocorrelated rates models, using our dR40 calibration set

\begin{tabular}{lllll}
\hline Clade & $\begin{array}{l}\text { Springer et al. [10] } \\
\text { minimum (Ma) }\end{array}$ & $\begin{array}{l}95 \% \text { CI (ind. } \\
\text { rates) (Ma) }\end{array}$ & $\begin{array}{l}95 \% \text { CI (auto. } \\
\text { rates) (Ma) }\end{array}$ & $\begin{array}{l}\text { Minimum } \\
\text { difference (Ma) }\end{array}$ \\
\hline Hyracoidea & 6.08 & $2.7-4.2$ & $2.9-4.6$ & 1.5 \\
Felis-Prionodon & 28.1 & $15.6-19.5$ & $20.8-25.3$ & 2.8 \\
Emballonuroidea & 47.8 & $35.5-40.4$ & $39.2-43.8$ & 4.0 \\
Erinaceidae-Soricidae & 61.6 & $53.5-57.4$ & $52.1-56.3$ & 4.2 \\
Lorisiformes & 38.0 & $13.3-17.4$ & $15.0-18.7$ & 19.3 \\
Lagomorpha & 53.7 & $37.3-44.0$ & $37.6-43.1$ & 9.7 \\
Aplodontidae- & 45.7 & $35.9-43.1$ & $39.9-44.2$ & 1.5 \\
Sciuridae & & & &
\end{tabular}

The minimum difference in age is between the fossil minimum and the oldest of the two $95 \%$ Cls. Differences $\geq 4.0$ are shaded grey. Each calibration minimum bound is poorly supported, as discussed in Additional file 1: "Incorrect or poorly supported fossil placements"

and some other Eocene European bats previously assigned to Emballonuridae and Rhinolophoidea have since been placed in a new family of uncertain affinities [37]. Tachypteron was not considered in that study. Ravel et al.'s [38] cladistic analysis of Tachypteron and Vespertiliavus included only emballonuroids among crown bats. Hence, the placement of Tachypteron requires confirmation, especially analysis of cranial and post-cranial material.

(4) The $~ 62 \mathrm{Ma}$ Adunator (calibrating ErinaceidaeSoricidae) has been considered an erinaceomorph (e.g. [39]). However, this assignment is precarious; other work questions whether Adunator even falls within Lipotyphla. Novacek et al. [40] stated that Adunator is in "limbo between primitive insectivorans and primitive condylarths", while [41] instead place Adunator with elephant shrews. Moreover, the calibration reference (A. ladae) has not been tested in formal phylogenetic analyses, and has recently been placed a resurrected genus (Mckennatherium) with even less certain affinities [42].

The potential for interordinal divergences to be inflated by poorly-justified calibration minimum bounds (or by rate model errors) is exacerbated by overly conservative maximum bounds. Maximum bounds should cover the time back until relatively well sampled fossil assemblages in potential geographic regions of origin that contain no putative crown group members, but contain stem members or ecological equivalents $[13,43]$ . These conditions are met for Chiroptera in the Thanetian (base $=59.2 \mathrm{Ma}$ ) [44] and for both Primates and
Rodentia in the Selandian (base $=61.6 \mathrm{Ma}$ ) [11]. Springer et al. [10] extend each of these maximum bounds by one or more additional geological stages, based on arbitrary phylogenetic bracketing from [45] or unspecified uncertainty in the case of bats, from [46].

It is now apparent that the few most problematic minimum and maximum calibration bounds listed above are the main drivers for Springer et al. [10] pushing the primary diversification of placental mammals back into the Cretaceous. This can be shown by starting with our dR40 analysis of the 122-taxon dataset, and then substituting in Springer et al.'s [10] minimum bounds for Lorisiformes, Lagomorpha, Emballonuroidea and Erinaceidae-Soricidae, and their maximum bounds for basal rodents, primates and bats. With this $\mathrm{dR} 40_{\text {Springer }}$ calibration set, the midpoint of the primary placental interordinal diversification shifts from 64.5 Ma, back to 72.2 Ma (Fig. 3), closely approaching Springer et al.'s [10] 74.1 Ma diversification midpoint. In contrast, our basal Paleocene estimate is in phase with the primary diversification in the fossil record (Fig. 1) and with a new generation of morphological/total evidence dating $[47,48]$.

Applying Springer et al.'s [10] more conservative rodent, primate and bat maximum bounds alone explains very little of the date increases shown in Fig. 3b. Substituting these older maxima into our dR40 analyses lifts the midpoint of the primary placental interordinal diversification by only $0.8 \mathrm{Ma}$, to $65.3 \mathrm{Ma}$ (Additional file 2). It is only with the further inclusion of the poorly-vetted lorisiform, lagomorph, emballonuroid and erinaceid-soricid minimum bounds that the interordinal divergences are pushed far older (Fig. 3b).

We compared the specified soft bounds, joint marginal priors and posterior estimates for the above-noted key 
nodes (Fig. 4). The posterior 95\% CIs fit better with our preferred dR40 calibrations (above: orange marginal prior, purple/brown posterior CIs) than with the $\mathrm{dR} 40_{\text {Springer }}$ calibrations (below: grey marginal prior, green/black posterior CIs). For all of these key calibrations our dR40 posterior CIs fell within or largely overlap the joint marginal prior. Whereas, with Springer et al.'s [10] calibrations, the posterior $\mathrm{CI}$ for either the independent or autocorrelated rates analysis falls fully outside both the specifed and marginal priors for Primates, Rodentia, Lagomorpha, Lorisiformes, and almost for Emballonuroidea. The lorisiform mismatch is extreme, being far younger than Springer et al.'s [10] Saharagalago reference fossil, which in turn, induces an anomalous 4.69-fold rate deceleration for this clade (see Additional file 2).

One recent genome-scale inference of mammal divergence [17] is remarkably sensitive to rate model assumptions, such that their results are difficult to place on Fig. 1. Even for their favoured STAR tree and gene-wise partitioning for MCMCtree, the primary interordinal diversification midpoint varies from $68.0 \mathrm{Ma}$ with independent rates to $94.7 \mathrm{Ma}$ with autocorrelated rates. Further substantial dating differences across partitioning schemes might also raise possible concerns about the underlying data (also see [49]), but two other issues are worth considering within our present context. Liu et al. [17] calibrate several very large, long-lived mammal clades, and 16 of 19 maximum bounds were defined by the presence of a stem lineage taxon (often the oldest, and with variously putative to well-agreed support). Maximum bounds should never be based on a specific fossil taxon - a practice that cannot account for sampling artefacts, and yet, when there is a good fossil record, can depend less on the age of the crown group being calibrated and more on the divergence from its sister taxon.

We employed Liu et al.'s [17] genomic data with our $\mathrm{dR} 40$ calibration scheme on the relevant nodes after deleting the large, long-lived taxa. The resulting timetrees (Additional file 2) provide far closer agreement between independent and autocorrelated rates models. Liu et al. [17] favoured the independent rates model over autocorrelated rates, based on several simulated and empirical tests. Under the independent rates model the placental diversification midpoint is $63.2 \mathrm{Ma}$, and the initial divergences within Laurasiatheria, Euarchontoglires and Afrotheria closely co-occur (all within $2.3 \mathrm{Ma}$ ) instead of being spread over 7.6 Ma as in Liu et al. [17]. Hence, with more rigorous calibration and reducing the potential for error-shift inflation, genome-scale data support the soft explosive model of placental evolution.

\section{Molecular rates and life history traits among early placental mammals}

Focusing calibration on clades that maintain ancestral evolutionary rates (or life history rate correlates) is the critical element shared by our most accurate dates for the simulated data and our empirical estimates for a placental origin younger than $80 \mathrm{Ma}$ and major diversification near
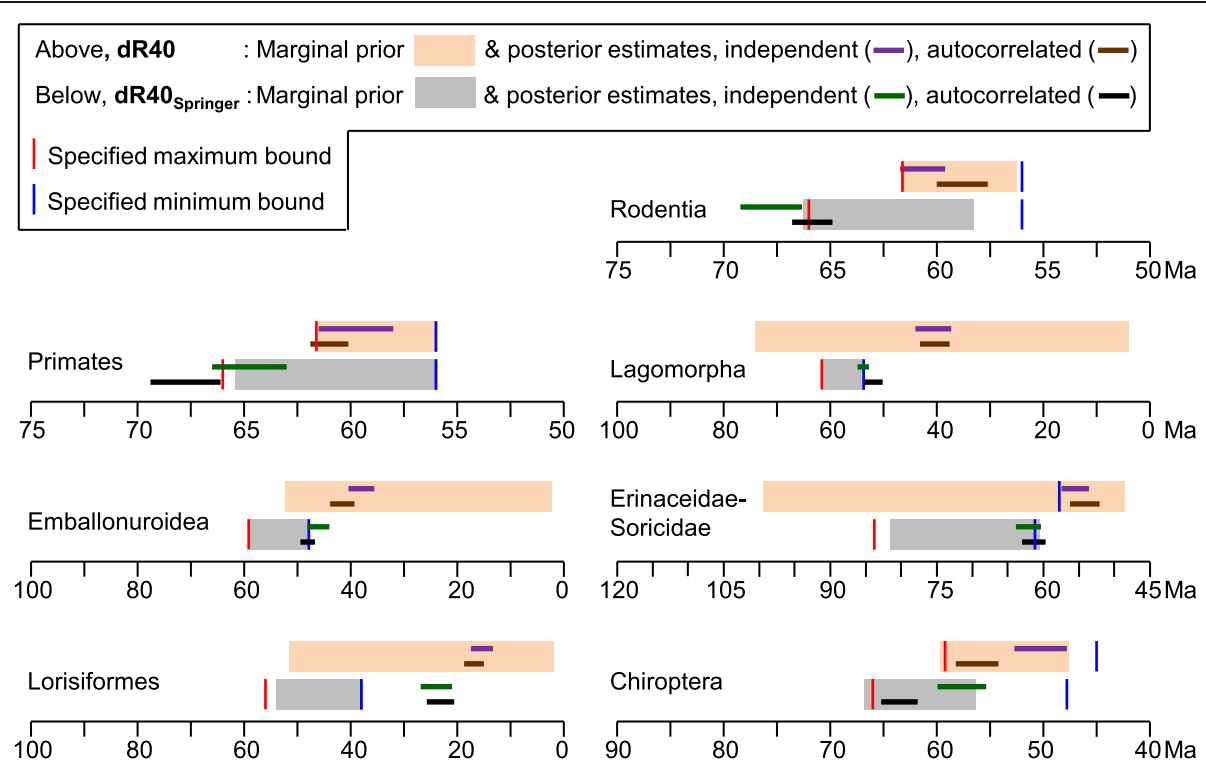

Fig. 4 Comparison of the dR40 and dR40 springer calibration bounds, joint marginal priors, and posterior divergence estimates for seven key clades. For each clade the calibration bounds and 95\% Cls for marginal priors and posterior estimates are shown (above) for our preferred dR40 calibration set, and (below) for the $\mathrm{dR}_{4} \mathrm{~S}_{\text {springer }}$ calibration set with calibration bounds substituted in from Springer et al. [10]. Posterior estimates are shown separately for the autocorrelated and independent rates models, however, the marginal priors under these two rates models are effectively the same, and here for clarity we average over the slight, primarily stochastic differences between them. (marginal priors and posterior estimates are provided in Additional file 2). Erinaceidae-Soricidae is further discussed in Additional file 1 ("Incorrect or poorly supported fossil placements", 7) 
the $66 \mathrm{Ma} \mathrm{KPg} \mathrm{event} \mathrm{(Fig.} \mathrm{2).} \mathrm{This} \mathrm{finding} \mathrm{was} \mathrm{foresha-}$ dowed by Phillips [11] showing that molecular rates for placental, marsupial and monotreme stem lineages were reliably traced back into the Mesozoic when calibrating clades that retain inferred ancestral life history traits, whereas calibrating only among large, long-lived mammals resulted in implausibly old divergences.

Inference of life history rate correlates from fossils also predicts that early eutherians had at least moderate rates of molecular evolution. All of the thousands of eutherian fossils from the period (Albian-Campanian: 113-72.1 Ma) that covers nearly all molecular date estimates for the origin and subsequent interordinal diversification of placentals were from small animals $(<250 \mathrm{~g}$ adult body mass) $[50,51]$. Lifespans of these extinct eutherians were also likely to have been relatively short, because maximum longevity among all similarly small modern, non-volant and non-fossorial placentals is less than 20 years (mean 7.2 years; 95\% CI 2.7-17.9 years, AnAge Database [52]).

One molecular argument against short longevity and high molecular rates among early placentals needs to be addressed. Romiguier et al. [18] analysed genomic protein coding GC content at 3rd positions (GC3) and found a remarkable correlation between GC3 conservation and longevity. They estimated maximum longevity of $25.7-40.9$ years for early placentals, which is well beyond the range noted above for modern eutherians that are as small as their Albian-Campanian counterparts. However, Romiguier et al.'s [18] GC3 conservation metric is a function of time since divergence, and they assumed that crown placentals originated at $105 \mathrm{Ma}$.

Romiguier et al. [18] presented a time-correlated index of GC3 conservation, $\gamma=-t / \log (\tau)$, where $t$ is time since divergence and $\tau$ is Kendall's correlation coefficient for GC3 conservation among genes, between species. We recalculated $\gamma$ for each of Romiguier et al.'s [18] GC3 conservation coefficients $(\tau)$ for taxon pairs, but with divergence estimates from Phillips [11]. We confirm the strong correlation between $\gamma$ and maximum longevity $\left(R^{2}=0.91\right.$; maximum longevity $=0.0683 \gamma-10.243$ ). This relationship allows divergence estimates for the origin of placental mammals to be cross validated against life history inferences drawn from the fossil record. If we use our mean $77 \mathrm{Ma}$ estimate for the placental origin from the $\mathrm{dR} 32 / \mathrm{dR} 40$ analyses, the maximum longevity estimate for early placentals falls dramatically, to 7.921.9 years (Additional file 4), and is now consistent with many modern, small placentals. Thus, the emerging picture is of placental mammals with size and longevity similar to tree shrews, inheriting the post-KPg world and rapidly diversifying into the ecospace opened up by the extinction of dinosaurs and many other land vertebrates.

\section{Molecular dating adaptive radiations}

O'Leary et al. [5] argue for both the origin and major diversification of crown placentals at the $\mathrm{KPg}$, but they did not account for fossil sampling errors. Unlike for some divergences within or between placental orders, unambiguous apomorphies for the placental crown are unclear, and biogeography reconstructions [6] give substantial probability to their origins being outside well-sampled Cretaceous mammal fossil faunas (e.g. in Africa). So we should not expect a close match between molecular estimates for the placental crown age and their earliest fossil records. Yet, the KPg molecular evolutionary rate spike that Springer et al. [29] claim for explosive models was produced by forcing this "hard" explosive model, which compresses the placental origin and $>15 \mathrm{Ma}$ of evolution on our "soft" explosive tree (Fig. 3a) into just 200,000 years - an extreme scenario that they dismissed (but see [53]). In contrast, our "soft" explosive model places the placental origin at $\sim 76-81 \mathrm{Ma}$ and the subsequent interordinal diversification (not the placental origin) near the $\mathrm{KPg}$, and molecular rate estimates for placentals remain much the same across the KPg [11]. However, parallel rate slowdowns occur in large-bodied, long-lived clades, such as whales and seacows [11], which upon calibration provide strong upwards pressure on interordinal divergences. Similar rate-shift inflation may be promoted in birds by parallel rate slowdowns, for example, among penguins and tubenoses [54].

We expect that the three key elements of error-shift inflation will often be associated with adaptive radiations. The first is that evolutionary races into novel ecospaces, which involve negotiating complex fitness landscapes, will favour species with large effective population sizes and high substitution rates [55-57], and these will typically be smaller, shorter-lived species. Much the same is predicted by theory around Cope's rule $[58,59]$ for the tendency for radiations to proceed from smaller to larger body size. The second element, is that once large body size does evolve, fossil sampling improves [60] and allometry drives apomorphy [61]. These factors tend to promote tighter minimum bounds, which combined with the rate deceleration concomitant with large body size, provides the basis for error-shift inflation. The third factor that is typical for adaptive radiations is that maximum bounds are often necessarily conservative for calibrations deeper in the tree, if they rely on detecting smaller, more plesiomorphic taxa. This in turn reduces the effectiveness of these maximum bounds for buffering against error-shift inflation associated with underestimation of parallel rate deceleration among large, long-lived taxa.

\section{Conclusions}

In the present study our simulations based on empirical rate variation show that error-shift inflation associated 
with parallel rate deceleration among large, long-lived placentals can explain the proposed overestimation of interordinal divergences among molecular dating analyses. We have overcome error-shift inflation by focusing taxon sampling (or calibration) on mammals with more plesiomorphic life history rate-correlates, and by reducing asymmetrical confidence in assigning minimum and maximum calibration bounds. As a result, the most profound diversification event in placental mammal history is brought into temporal agreement between molecular dates and the fossil record (Fig. 1). A similar soft explosive model of diversification immediately following the KPg is now emerging among birds, within both Neoaves [19, 54, 62] and palaeognaths [63]. Better understanding the relationship between natural history rate-correlates and calibration strategies may be important for resolving molecular dating/fossil record controversies for other adaptive radiations, such as for the Cambrian explosion of metazoans, and for flowering plants.

\section{Methods \\ Simulating molecular rate evolution and error-shift inflation among placental mammals}

We simulated mammalian molecular data to understand whether realistic patterns of molecular rate variation, including parallel rate decelerations among large, long-lived taxa could explain interordinal divergence overestimates, when controlling for calibration. For each set of simulations we used Seq-Gen 1.3.3 [64] to generate 100 datasets of 20,000 bp sequences for a 12-taxon phylogeny (Fig. 2a) that is simplified from the proposed mammalian timetree of Phillips [11]. In addition to "monotreme" and "marsupial" outgroups, the "placental" ingroup has its crown origin at $80 \mathrm{Ma}$, with two daughter nodes at $66 \mathrm{Ma}$ (mimicking superordinal divergences, such as Laurasiatheria and Afrotheria), and each splitting into two $33 \mathrm{Ma}$ clades.

In the first set of simulations, which we refer to as "ancestral rates", the branch rates are randomly drawn from a lognormal distribution (ln mean-6.523, s.d. 0.274) modelled on inferred rates for small to mid-sized mammals ( $<30 \mathrm{~kg}$ adult body mass, $<40$ years maximum longevity) from [11], based on the 26-gene, 169-taxon dataset of Meredith et al. [2]. A second set of simulations that we refer to as "mixed rates" draws from the "ancestral rates" distribution for most of the tree, but mimics large, long-lived taxa for two 33 Ma clades diverging from nodes I and III in Fig. 2. These rates are drawn from the same lognormal distribution, but scaled to $1 / 5$, similar to whales or seacows, from Phillips [11].

Timetrees for each simulated dataset were inferred separately in MCMCtree [25, 26], using the independent rates model. Calibrations were all symmetric, with $2.5 \%$ soft bound minima and maxima equidistant from the "true" simulated age. These age bounds are shown in Fig. $2 \mathrm{~b}$ for all calibrated nodes. The simulated datasets were analysed either with all six calibrations (full calibration) or without calibrating the two clades (I \& III) that exhibit the rate deceleration (ancestral rate nodes calibration).

\section{Empirical data and deleting large, long-lived taxa}

Mammalian timetrees were estimated from two DNA datasets based on the 26-gene (35,603 bp), 169-taxon alignment of Meredith et al. [2]. The first is the 122-taxon dataset, for which Springer et al. [10] had deleted all taxa included by Meredith et al. [2] that are $>10 \mathrm{~kg}$ and/or $>$ 40 years maximum longevity. The second dataset (128 taxa) includes additional mammals up to $30 \mathrm{~kg}$, to test the sensitivity of the date estimates to including medium sized mammals well outside the upper size bound of any Mesozoic eutherians, but that are not especially long lived (still $<40$ years maximum longevity). In addition, we estimated timetrees from Liu et al.'s [17] three favoured "first quintile" 200-gene alignments, again including only the 57 taxa that are $<10 \mathrm{~kg}$ and $<40$ years maximum longevity.

The fossil calibration bounds employed for each of the empirical timetree analyses are provided in Additional file 3, and joint marginal priors are provided in Additional file 2. To summarize, our initial analysis of the dataset for 122 taxa with presumed plesiomorphic life histories employs the calibration scheme of Phillips [11], except for calibrations on nodes deleted by Springer et al. [10]. Most placental mammal calibrations were originally based on [12]. Next we added eight additional, well-supported calibrations, including from among those that Springer et al. [10] implied would increase our divergence estimates, lifting the number of calibrations to 40. A further three calibrations were added upon the inclusion of additional taxa up to $30 \mathrm{~kg}$ for the 128-taxon dataset. Twenty-four of our favoured calibrations were compatible with the taxon sampling for the alignments derived from Liu et al. [17].

\section{Geomolecular dating with MCMCtree}

All timetrees based on empirical and simulated data were inferred with MCMCtree, within PAML [28]. Both the independent rates and autocorrelated rates models were employed for the 122-taxon and 128-taxon empirical datasets, with control-file priors and run parameters replicating Springer et al. [10]. This includes unit time $(100 \mathrm{Ma})$, the rate prior parameters, rgene_gamma shape (1) and scale (5.41), and the rate drift prior parameters, sigma_gamma shape (1) and scale (4.207). Analyses were run for 200,000 generations, sampled every 50th generation, and a burnin of 10,000 generations was discarded. The 57-taxon alignments were similarly run in MCMCtree, although matching the original priors used 
by Liu et al. [17], including root age (4.16-4.254 Ma) and rgene_gamma $(2,40)$.

Rate distributions for the simulated datasets were based on the empirical data of Meredith et al. [2], with rate estimates taken from Phillips (Figure five in [11]; also see "Results and Discussion"). In the case of the "ancestral rates" simulations, rates were modelled only from branches representing mammals $<30 \mathrm{~kg}$ adult body mass and $<40$ years maximum longevity. Given the rate estimate for these data of 0.1469 subs per $100 \mathrm{Ma}$, the rgene_gamma scale parameter was adjusted to $6.81(=1 /$ 0.1469 ). The "mixed rates" analyses include four $33 \mathrm{Ma}$ branches with $1 / 5$ the ancestral rate, and as such the rgene_gamma scale parameter was adjusted to 7.51. The root age prior for all analyses of simulated data was $200 \mathrm{Ma}$ (with sigma_gamma scale 2.0), with the root being symmetrically calibrated with soft $2.5 \%$ prior minimum and maximum bounds from 180 to $220 \mathrm{Ma}$.

\section{Eutherian mammal diversification in the fossil record}

Direct reading of the eutherian fossil record implies an extraordinary diversification immediately following the $66 \mathrm{Ma} \mathrm{KPg}$ event $[7,8,11]$. However, Springer et al. [10] advocate other diversification spikes well before the $\mathrm{KPg}$, during the Turonian (93.9-89.3 Ma) and Campanian (83.6-72.1 Ma). They also suggest another diversification spike after the KPg, during the Ypresian (56.0-47.8 Ma). However, it is important to consider fossil sampling. A stage with a short duration and poor sampling will artefactually appear to have few new species appearances, while the same actual diversification rate will result in many more new species appearances for a longer, and better sampled stage, especially if it follows a stage in which new appearances were masked by poor sampling.

We obtained fossil species richness and new appearance counts from The Paleobiology Database (accessed 29 March 2017). To help even out sampling potential we start with the critical (and well-sampled) Campanian, Maastrichtian and Paleocene, and then provide further time bins as individual or combined stages that sample at least 80 mammal species. Mammals overall provide a better indicator of sampling potential than eutherians, which are expected to have very low species richness close to their origin. Our strategy resulted in relatively even bin durations (average 8.78 Ma, s.d. 2.17 Ma, see Fig. 1, Additional file 5), except for the oldest bin, Albian-Cenomanian (19.1 Ma duration), which is outside the range of molecular and morphological predictions for the diversification spike. A second important factor that Springer et al. [10] did not consider for either fossil or molecular diversification analysis is the standing diversity base from which new fossil appearances derive, or from which new molecular lineages diverge, as is standard in lineage through time analysis (see $[16,65]$ ).
Our metric for eutherian diversification is the number of new eutherian species appearances for the time bin, divided by both the duration of the time bin and the standing diversity of eutherians in the previous time bin. Fossil sampling potential is still unlikely to be constant across all of the time bins. Therefore, to integrate out much of the sampling disparity we also show new eutherian appearances in each bin as a proportion of new appearances among all mammals (indicated by colour scaling in Fig. 1, also see Additional file 5).

\section{Additional files}

Additional file1: Addressing claims of "zombie" lineages on Phillips' (2016) timetree. Table S1. and Figure S1. (PDF 588 kb)

Additional file 2: MCMCtree timetrees. Table S2. Figure S2. and Figure S3. (PDF $525 \mathrm{~kb}$ )

Additional file 3: Fossil calibration schemes. Table S3. and Figure S4. (PDF 583 kb)

Additional file 4: GC3 conservation and estimating maximum longevity. Figure S5. (PDF $251 \mathrm{~kb}$ )

Additional file 5: Table S4. Fossil record species richness for Eutheria and Mammalia from Albian through to Lutetian. (PDF $90 \mathrm{~kb}$ )

\section{Abbreviations \\ Cl: Credible interval; KPg: Cretaceous-Paleogene boundary; Ma: Mega-anna (millions of years)}

\section{Acknowledgements}

We are grateful to David Penny and Peter Waddell for valuable discussions on mammalian diversification and to Robin Beck for helpful comments on the manuscript. Thomas Halliday kindly provided dated morphological trees, and Adam Stone assisted with QUT High Performance Computing. Three anonymous reviewers provided valuable comments that improved the manuscript.

\section{Funding}

This research was funded by an Australian Research Council Discovery grant (DP150104659 to MJP).

\section{Availability of data and materials}

All data analysed during this study are included in this published article and its supplementary information files, or appropriately cited and publically available from earlier studies.

\section{Authors' contributions}

MJP designed the study, curated the fossil calibrations, simulated DNA data, ran the fossil record and longevity analyses, and wrote the manuscript. CF ran the MCMCtree analyses and collated published molecular dates. Both authors read and approved the final manuscript.

Ethics approval and consent to participate

Not applicable.

Consent for publication

Not applicable.

Competing interests

The authors declare that they have no competing interests.

\section{Publisher's Note}

Springer Nature remains neutral with regard to jurisdictional claims in published maps and institutional affiliations. 


\section{Received: 17 October 2017 Accepted: 19 June 2018}

\section{Published online: 03 July 2018}

\section{References}

1. Bininda-Emonds ORP, Cardillo M, Jones KE, MacPhee RDE, Beck RMD, Grenyer R, Price SA, Vos RA, Gittleman JL, Purvis A. The delayed rise of present-day mammals. Nature. 2007;446(7135):507-12.

2. Meredith RW, Janečka JE, Gatesy J, Ryder OA, Fisher CA, Teeling EC, Goodbla A, Eizirik E, Simão TLL, Stadler T, et al. Impacts of the cretaceous terrestrial revolution and $\mathrm{KPg}$ extinction on mammal diversification. Science. 2011;334(6055):521-4.

3. Tarver JE, dos Reis M, Mirarab S, Moran RJ, Parker S, O'Reilly JE, King BL, O'Connell MJ, Asher RJ, Warnow T, et al. The interrelationships of placental mammals and the limits of phylogenetic inference. Genome Biol Evol. 2016; 8(2):330-44.

4. Wible JR, Rougier GW, Novacek MJ, Asher RJ. The eutherian mammal Maelestes gobiensis from the late cretaceous of Mongolia and the phylogeny of cretaceous Eutheria. Bull Am Mus Nat Hist. 2009;316:1-123.

5. O'Leary MA, Bloch Jl, Flynn JJ, Gaudin TJ, Giallombardo A, Giannini NP Goldberg SL, Kraatz BP, Luo ZX, Meng J, et al. The placental mammal ancestor and the post-K-Pg radiation of placentals. Science. 2013; 339(6120):662-7.

6. Springer MS, Meredith RW, Janecka JE, Murphy WJ. The historical biogeography of Mammalia. Phil Trans Roy Soc B. 2011:366(1577):2478-502.

7. Alroy J. The fossil record of north American mammals: evidence for a Paleocene evolutionary radiation. Syst Biol. 1999;48(1):107-18.

8. Longrich NR, Scriberas J, Wills MA. Severe extinction and rapid recovery of mammals across the cretaceous-Palaeogene boundary, and the effects of rarity on patterns of extinction and recovery. J Evol Biol. 2016;29(8):1495-512

9. Bininda-Emonds ORP, Purvis A. Comment on "impacts of the cretaceous terrestrial revolution and $\mathrm{KPg}$ extinction on mammal diversification". Science. 2012;337(6090):34.

10. Springer MS, Emerling CA, Meredith RW, Janečka JE, Eizirik E, Murphy WJ. Waking the undead: implications of a soft explosive model for the timing of placental mammal diversification. Mol Phylogenet Evol. 2017;106:86-102.

11. Phillips MJ. Geomolecular dating and the origin of placental mammals. Syst Biol. 2016;65(3):546-57.

12. dos Reis M, Inoue J, Hasegawa M, Asher RJ, Donoghue PCJ, Yang Z Phylogenomic datasets provide both precision and accuracy in estimating the timescale of placental mammal phylogeny. Proc Roy Soc B. 2012; 279(1742):3491-500.

13. Parham JF, Donoghue PCJ, Bell CJ, Calway TD, Head JJ, Holroyd PA, Inoue JG, Irmis RB, Joyce WG, Ksepka DT, et al. Best practices for justifying fossil calibrations. Syst Biol. 2012;61(2):346-59.

14. Benton MJ. Early origins of modern birds and mammals: molecules vs. morphology. Bioessays. 1999;21(12):1043-51.

15. Simpson GG. The principles of classification and a classification of mammals. Bull Am Mus Nat Hist. 1945;85:1-307.

16. Foote M, Hunter JP, Janis CM, Sepkoski JJ. Evolutionary and preservational constraints on origins of biologic groups: divergence times of eutherian mammals. Science. 1999;283(5406):1310-4.

17. Liu L, Zhang J, Rheindt FE, Lei FM, Qu YH, Wang Y, Zhang Y, Sullivan C, Nie WH, Wang $J$, et al. Genomic evidence reveals a radiation of placental mammals uninterrupted by the KPg boundary. Proc Natl Acad Sci U S A. 2017;114(35):E7282-90

18. Romiquier J, Ranwez V, Douzery EJP, Galtier N. Genomic evidence for large, long-lived ancestors to placental mammals. Mol Biol Evol. 2013;30(1):5-13.

19. Jarvis ED, Mirarab S, Aberer AJ, Li B, Houde P, Li C, Ho SYW, Faircloth BC, Nabholz B, Howard JT, et al. Whole-genome analyses resolve early branches in the tree of life of modern birds. Science. 2014;346(6215):1320-31.

20. Mitchell KJ, Cooper A, Phillips MJ. Comment on "whole-genome analyses resolve early branches in the tree of life of modern birds". Science. 2015; 349(6255):1460.

21. Beaulieu JM, O'Meara BC, Crane P, Donoghue MJ. Heterogeneous rates of molecular evolution and diversification could explain the Triassic age estimate for angiosperms. Syst Biol. 2015;64(5):869-78.

22. Brown JW, Smith SA. The past sure is tense: on interpreting phylogenetic divergence time estimates. Syst Biol. 2018;67:340-53.

23. Foster CSP, Sauquet $H_{1}$ Van der Merwe M, McPherson $H$, Rossetto $M, H o$ SYW. Evaluating the impact of genomic data and priors on Bayesian estimates of the angiosperm evolutionary timescale. Syst Biol. 2017;66(3): $338-51$

24. Lee MSY, Soubrier J, Edgecombe GD. Rates of phenotypic and genomic evolution during the Cambrian explosion. Curr Biol. 2013:23(19):1889-95.

25. Yang Z. PAML 4: phylogenetic analysis by maximum likelihood. Mol Biol Evol. 2007:24(8):1586-91.

26. Yang Z, Rannala B. Bayesian estimation of species divergence times under a molecular clock using multiple fossil calibrations with soft bounds. Mol Biol Evol. 2006;23(1):212-26.

27. Lane A, Janis CM, Sepkoski JJ. Estimating paleodiversities: a test of the taxic and phylogenetic methods. Paleobiology. 2005;31(1):21-34.

28. Springer MS, Burk-Herrick A, Meredith R, Eizirik E, Teeling E, O'Brien SJ, Murphy WJ. The adequacy of morphology for reconstructing the early history of placental mammals. Syst Biol. 2007;56(4):673-84.

29. Springer MS, Meredith RW, Teeling EC, Murphy WJ. Technical comment on "the placental mammal ancestor and the post-K-Pg radiation of placentals". Science. 2013:341(6146):613.

30. Herrera JP, Dávalos LM. Phylogeny and divergence times of lemurs inferred with recent and ancient fossils in the tree. Syst Biol. 2016;65(5):772-91.

31. Marivaux L, Ramdarshan A, Essid EM, Marzougui W, Ammar HK, Lebrun R, Marandat B, Merzeraud G, Tabuce R, Vianey-Liaud M. Djebelemur, a tiny pretooth-combed primate from the Eocene of Tunisia: a glimpse into the origin of crown strepsirhines. PLoS One. 2013;8(12):e80778.

32. Rose KD, DeLeon VB, Missiaen P, Rana RS, Sahni A, Singh L, Smith T. Early Eocene lagomorph (Mammalia) from western India and the early diversification of Lagomorpha. Proc Roy Soc B. 2008;275(1639):1203-8.

33. Matthee CA, van Vuuren BJ, Bell D, Robinson TJ. A molecular supermatrix of the rabbits and hares (Leporidae) allows for the identification of five intercontinental exchanges during the Miocene. Syst Biol. 2004:53(3):433-47.

34. Zhang Z, Li C, Wang J. Presence of the calcaneal canal in basal Glires. Vertebrata PalAsiatica. 2016;54(3):235-42.

35. Storch G, Sigé B, Habersetzer J. Tachypteron franzeni n. Gen., n. Sp., earliest emballonurid bat from the middle Eocene of Messel (Mammalia, Chiroptera). Paläontol Z. 2002;76(2):189-99.

36. Teeling EC, Springer MS, Madsen O, Bates P, Brien SJ, Murphy WJ. A molecular phylogeny for bats illuminates biogeography and the fossil record. Science. 2005;307:580-4.

37. Maitre E, Sigé B, Escarguel G. A new family of bats in the Paleogene of Europe: systematics and implications for the origin of emballonurids and rhinolophoids. Neues Jahrbuch fur Geologie und PalaontologieAbhandlungen. 2008;250(2):199-216.

38. Ravel A, Adaci M, Bensalah M, Charruault A-L, Essid EM, Ammar HK, Marzougui W, Mahboubi M, Mebrouk F, Merzeraud G, et al. Origine et radiation initiale des chauves-souris modernes: nouvelles découvertes dans I'Éocène d'Afrique du Nord. Geodiversitas. 2016:38(3):355-434.

39. Brown TM, Schankler D. A review of the Proteutheria and Insectivora of the Willwood formation (lower Eocene) Bighorn Basin, Wyoming. U S Geol Surv Bull. 1982;1523:1-79.

40. Novacek MJ, Bown TM, Schankler DM. On the classification of early tertiary Erinaceomorpha (Insectivora, Mammalia). Am Mus Novit. 1985;2813:1-22.

41. Hooker JJ, Russell DE. Early Palaeogene Louisinidae (Macroscelidea, Mammalia), their relationships and north European diversity. Zool J Linnean Soc. 2012:164(4):856-936.

42. Secord R. The Tiffanian land-mammal age (middle and late Paleocene) in the northern Bighorn Basin, Wyoming. Univ Mich Pap Paleontology. 2008;35:1-192

43. Barnett R, Barnes I, Phillips MJ, Larry D, Martin C, Leonard JA, Cooper A. Evolution of the extinct sabretooths and the American cheetah-like cat. Proc Natl Acad Sci U S A. 2005;101:1626-31.

44. Phillips MJ. Four mammal fossil calibrations: balancing competing palaeontological and molecular considerations. Palaeontol Electron. 2015; 18(1):1-16.

45. Benton MJ, Donoghue PC, Asher RJ, Friedman M, Near TJ, Vinther J. Constraints on the timescale of animal evolutionary history. Palaeontol Electron. 2015:18(1):1-106.

46. Emerling CA, Huynh HT, Nguyen MA, Meredith RW, Springer MS. Spectral shifts of mammalian ultraviolet-sensitive pigments (short wavelengthsensitive opsin 1) are associated with eye length and photic niche evolution. Proc Roy Soc B. 2015;282:2015817.

47. Halliday TJD, Upchurch P, Goswami A. Eutherians experienced elevated evolutionary rates in the immediate aftermath of the cretaceous Palaeogene mass extinction. Proc Roy Soc B. 2016;283:20153026. 
48. Ronquist F, Lartillot N, Phillips MJ. Closing the gap between rocks and clocks using total-evidence dating. Phil Trans Roy Soc B. 2016;371:20150136.

49. Gatesy J, Springer MS. Phylogenomic red flags: homology errors and zombie lineages in the evolutionary diversification of placental mammals. Proc Natl Acad Sci U S A. 2017;114:E9431-2.

50. Slater GJ. Phylogenetic evidence for a shift in the mode of mammalian body size evolution at the cretaceous-Palaeogene boundary. Methods Ecol Evol. 2013;4(8):734-44.

51. Halliday TJD, Goswami A. The impact of phylogenetic dating method on interpreting trait evolution: a case study of cretaceous-Palaeogene eutherian body-size evolution. Biol Lett. 2016;12:20160051.

52. de Magalhaes JP, Costa J. A database of vertebrate longevity records and their relation to other life-history traits. J Evol Biol. 2009;22(8):1770-4

53. Beck RMD, Lee MSY. Ancient dates or accelerated rates? Morphological clocks and the antiquity of placental mammals. Proc Roy Soc B. 2014;281: 20141278.

54. Ksepka DT, Phillips MJ. Avian diversification patterns across the K-Pg boundary: influence of calibrations, datasets, and model misspecification. Ann Mo Bot Gard. 2015;100:300-28.

55. Gavrilets S, Vose A. Dynamic patterns of adaptive radiation. Proc Natl Acad Sci U S A. 2005;102:18040-5.

56. Charlesworth B. Effective population size and patterns of molecular evolution and variation. Nat Rev Genet. 2009;10:195-205.

57. Lynch M, Abegg A. The rate of establishment of complex adaptations. Mo Biol Evol. 2010;27(6):1404-14.

58. Rensch B. Histological changes correlated with evolutionary changes of body size. Evolution. 1948;2(3):218-30.

59. Polly PD. Cope's rule. Science. 1998;282(5386):47.

60. Kidwell SM, Flessa KW. The quality of the fossil record: populations, species, and communities. Annu Rev Ecol Syst. 1995;26(1):269-99.

61. Marroig G, Cheverud JM. Size as a line of least evolutionary resistance: diet and adaptive morphological radiation in new world monkeys. Evolution. 2005;59(5):1128-42.

62. Prum RO, Berv JS, Dornburg A, Field DJ, Townsend JP, Lemmon EM, Lemmon AR. A comprehensive phylogeny of birds (Aves) using targeted next-generation DNA sequencing. Nature. 2015;526(7574):569-73.

63. Grealy A, Phillips M, Miller G, Gillbert MT, Rouillard JM, Lambert D, Bunce M, Haile J. Eggshell palaeogenomics: Palaeognath evolutionary history revealed through ancient nuclear and mitochondrial DNA from Madagascan elephant bird (Aepyornis sp.) eggshell. Mol Phylogenet Evol. 2017;109:151-63.

64. Rambaut A, Grassly NC. Seq-gen: an application for the Monte Carlo simulation of DNA sequence evolution along phylogenetic trees. Bioinformatics. 1997;13(3):235-8.

65. Rabosky DL. Likelihood methods for detecting temporal shifts in diversification rates. Evolution. 2006;60(6):1152-64.

66. Lartillot N, Phillips MJ, Ronquist F. A mixed relaxed clock model. Phil Trans Roy Soc B. 2016;371:20150132.

67. Murphy WJ, Pringle TH, Crider TA, Springer MS, Miller W. Using genomic data to unravel the root of the placental mammal phylogeny. Genome Res. 2007:17(4):413-21.

68. Springer MS, Murphy WJ, Eizirik E, O'Brien SJ. Placental mammal diversification and the cretaceous-tertiary boundary. Proc Natl Acad Sci U S A. 2003;100(3):1056-61.

69. Kumar S, Stecher G, Suleski M, Hedges SB. TimeTree: a resource for timelines, timetrees, and divergence times. Mol Biol Evol. 2017;34(7):1812-9.

\section{Ready to submit your research? Choose BMC and benefit from:}

- fast, convenient online submission

- thorough peer review by experienced researchers in your field

- rapid publication on acceptance

- support for research data, including large and complex data types

- gold Open Access which fosters wider collaboration and increased citations

- maximum visibility for your research: over $100 \mathrm{M}$ website views per year

At BMC, research is always in progress.

Learn more biomedcentral.com/submissions 